\title{
UPA for Image Transmission with Chaotic Communication
}

\author{
Monika Vyas ${ }^{1}$, Sumeet Dhillon ${ }^{2}$
}

\begin{abstract}
The infringement of remote correspondence inside the most recent couple of decades has brought about the advancement of various transmit and get (MIMO) frameworks empowering effective constant picture and video correspondence. These favorable circumstances of different radio wire frameworks close by productive transmission and coding plans might be investigated to accomplish very high information rates nearby enhanced information unwavering quality. Following the idea this paper displays a novel approach for transmitting pictures over an uproarious channel. The proposed strategy utilizes OFDM and riotous encoder plans to achieve twisting less and secure picture transmission inside the boisterous channel..Keywords: MIMO system, OFDM, chaos, unequal powerallocation, distortion.
\end{abstract}

Keywords: MIMO system, OFDM, chaos, unequal power allocation, distortion

\section{Introduction}

The enormous blast in the gadgets and remote correspondence industry has prompted progressing web office and economically accessible remote gadgets. Additionally utilization of pictures and recordings has expanded the information exchange by many folds. This has set the need of proficient and solid correspondence methods with information rates. To oblige these necessities late advances in the field of remote interchanges has prompted some surprising leaps forward in the area of multiuser spacetime remote correspondences.

The disclosure of the various info numerous yield (MIMO) remote frameworks indicates gigantic potential to assuage the bottleneck of remote information transmission. MIMO offers a development in movement limit with respect to future cell frameworks, to deal with the assignment of web serious applications. MIMO correspondence framework utilizes different recieving wires at the transmitter and the beneficiary that makes virtual spatial subchannels, over which various information streams can be transmitted. Each subchannel utilizes precisely the same, and the transmissions happen at the same time. Actually MIMO

frameworks can be considered as an augmentation of brilliant recieving wire frameworks, a most loved method, going back a very long while, for enhancing join dependability using radio wire exhibit bar shaping [1]. Additionally even the future $4 \mathrm{G}$ remote systems will join the capable advancements of MIMO, versatile and reconfigurable frameworks (programming radio) and remote access innovations such as orthogonal recurrence division various access (OFDMA) and numerous bearer code division different access (MC-CDMA) [2].

This paper shows a story thought of unequal power allotment plot for transmission of advanced pictures over a MIMO framework. The proposed framework utilizes the use of OFDM and disorganized correspondence to transmit the information pictures from various transmit reception apparatuses utilizing unequal transmit energy of the MIMO framework.

\section{Literature Survey}

Since 1990 s, a regularly expanding interest has been the use of tumultuous capacities to execute the encryption procedure. Tumultuous correspondence is an utilization of bedlam hypothesis to give secure transmission of information [3]. A turbulent framework in this manner can be named as a deterministic framework showing non-direct frameworks conduct with certain famous highlights. Such a framework utilizes the utilization of complex dynamic conduct, for example, pseudorandom clamor and spread range to encode information. Synchronization of disordered frameworks and its safe correspondence applications have been a noteworthy research territory in the course of the most recent decade [4, 5]. Pecora and Carroll, in their notable work [6] have proposed a stable turbulent subframework for building unidirectionally coupled synchronized frameworks.

Tumultuous specialized strategies, for example, riotous covering, turbulent move keying [7] and those on the premise of the projective synchronization [8] and stage synchronization [9] have likewise been broke down and proposed to be secure. In [10] a safe advanced correspondence framework in view of confused regulation, cryptography, and disorderly synchronization procedures have been proposed and investigated numerically. In [11], a route in light of encryption strategy is proposed. The proposed framework utilizes diverse yield from confused transmitter, transmitted in the channel as a keystream to scramble the message flag.

\section{Chaotic Encoder Formulation}

A general chaotic system can be described by following equation:

$$
\mathrm{x}^{\prime}=A \mathrm{x}+\mathrm{g}(\mathrm{x})
$$

where $\mathrm{Au}$ is the linear part, $\mathrm{g}(\mathrm{x})$ is the nonlinear part of the system. For this paper we construct chaotic system by using Lorenz's chaotic system which is an autonomous 3-order nonlinear system [12] and can be represented in following states: 


\section{International Journal of Science and Research (IJSR)}

ISSN (Online): 2319-7064

Index Copernicus Value (2016): 79.57 | Impact Factor (2015): 6.391

$$
\begin{aligned}
& \mathrm{x}_{1}{ }_{1}=A\left(\mathrm{x}_{2}-\mathrm{x}_{1}\right) \\
& \mathrm{x}_{2}{ }_{2}=\mathrm{c} \mathrm{x}_{1}-\mathrm{x}_{1} \mathrm{x}_{3}-\mathrm{x}_{2} \\
& \mathrm{x}_{3}{ }_{3}=\mathrm{x}_{1} \mathrm{x}_{2}-\mathrm{bx}_{3}
\end{aligned}
$$

where $\mathrm{a}, \mathrm{b}$, and $\mathrm{c}$ are constants greater than zero and $\mathrm{x}_{1}, \mathrm{x}_{2}$, and $\mathrm{x}_{3}$ are the dynamic states.

The input message $M$ is masked by the Encrpt and decrpt systems are presented as following:

Where $\mathrm{v}=\mathrm{u}_{1}+M$

$$
\text { Encrypt side(master): } \mathrm{x}^{\prime}=A \mathrm{x}+\mathrm{g}(\mathrm{x}, \mathrm{v})+\mathrm{Lz}_{\mathrm{x}}
$$

Decrypt side (slave): $\mathrm{y}^{\prime}=\mathrm{Ay}+\mathrm{g}(\mathrm{y}, \mathrm{v})+\mathrm{Lz} \mathrm{y}_{\mathrm{y}}$

chaotic state and transmitted. The equations of where $\mathrm{x} C$ $\mathrm{R}^{\mathrm{n}}, \mathrm{y} \in \mathrm{R}^{\mathrm{n}}$ are the state vectors. Ax and Ay are the linear part, $g(x, v)$ and $g(y, v)$ are the nonlinear part of this system, $\mathrm{L}$ is the controller gain of the system, $\mathrm{K}>0$ is the coupling strength between master and slave system, $\mathrm{z}_{\mathrm{x}}$ and $\mathrm{z}_{\mathrm{y}}$ are the feedback signal.

\section{Proposed System}

The Proposed framework utilizes the blend of OFDM (Orthogonal Frequency Multiple Access) and Chaotic Communication. Fig. 1 underneath indicates square chart of the transmission area of the proposed framework. The info picture is first changed over into bits and afterward these bits are given to various tumultuous encoder squares. The yield of the encoders is then given to the OFDM particular transmitters area. The OFDM yields are then transmitted utilizing the distinctive radio wires of the MIMO framework as appeared in Fig. 1. At the beneficiary side, the getting radio wires collector the transmitted picture duplicates. The gotten bits are first given to the OFDM collector, the yield of which is given to the confused decoder. The mean of the yields from the riotous decoders is figured the at long last changed over to give the last yield picture as in Fig. 2. Since the proposed procedure utilizes a MIMO framework the transmitted duplicates of pictures at various information control levels experience distinctive twisting through the channel. At the beneficiary these picture duplicates are decoded and a mean is computed to give the yield picture. This limits the mutilation in the got picture.

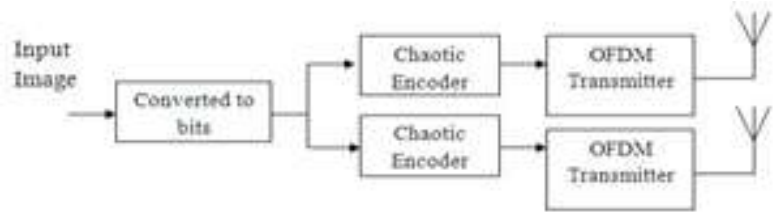

Figure 1: Transmitter Sec. of proposed System

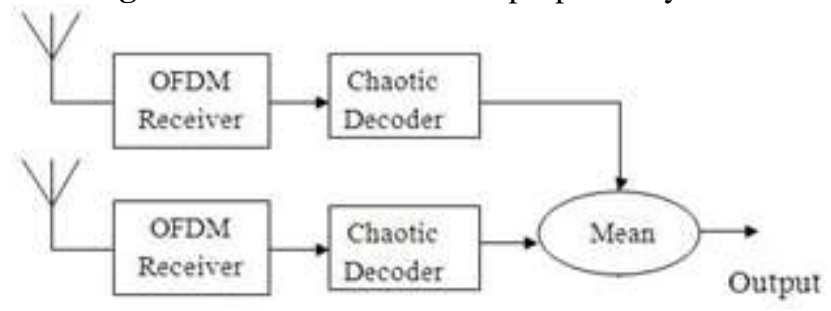

Figure 2: Receiver Section of proposed system

\section{Results}

The proposed show has been actualized in MATLAB. Fig 3 underneath demonstrates the info picture and Figure 4 demonstrates the yield of the OFDM transmitter pieces. Fig 5 demonstrates the BER v/s SNR chart for the proposed framework. From the diagram unmistakably the BER for the transmission of picture in Fig 3 is of the request of 10-6. Fig 6 demonstrates the deferral (in $\mathrm{ms}$ ) diagram for different SNR esteems. Fig 7 demonstrates the prepared information and the decoded yield pictures. Since the BER for the picture is less the yield picture is correct imitation of the info picture. The proposed framework is tried utilizing diverse pictures and it is support that the proposed framework has less BER when contrasted with straightforward transmission of the picture through a similar channel.

Table 1 beneath demonstrates the correlation of BER for transmission couple of standard pictures in Microsoft windows utilizing the proposed strategy utilizing a MIMO framework and through a basic single OFDM transmitter for a SNR of $30 \mathrm{~dB}$. From the table its is seen that the proposed technique has a superior BER for the SNR esteem when contrasted with basic transmission of a picture through single OFDM transmitter.

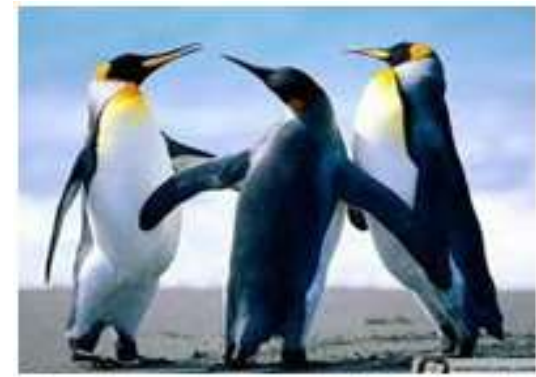

Figure 3: Receiver Section of proposed system OFDM 1 generated
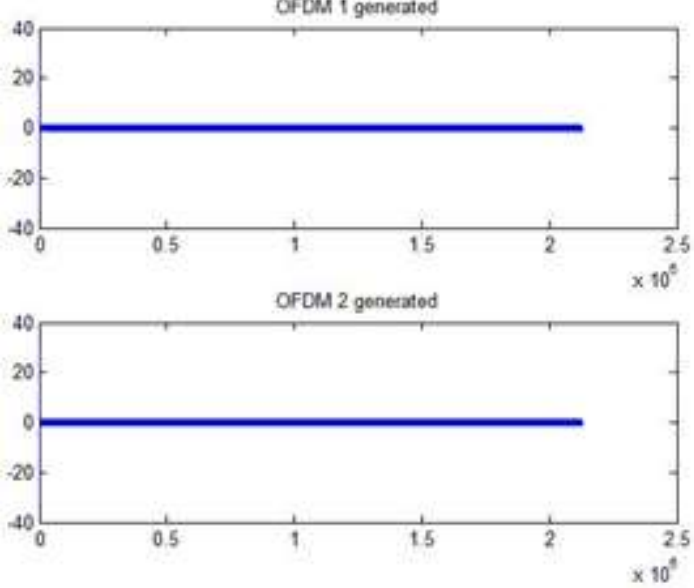

Figure 4: OFDM Transmitter output Proposed system 
International Journal of Science and Research (IJSR)

ISSN (Online): 2319-7064

Index Copernicus Value (2016): 79.57 | Impact Factor (2015): 6.391
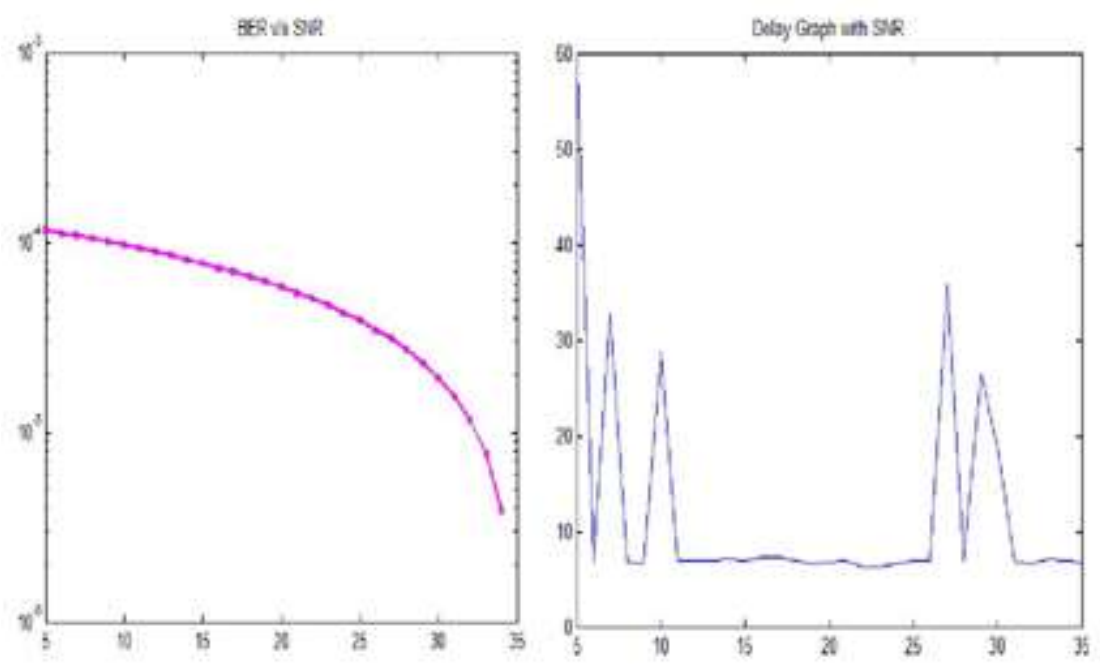

Figure 5: BER v/s SNR graph of proposed system

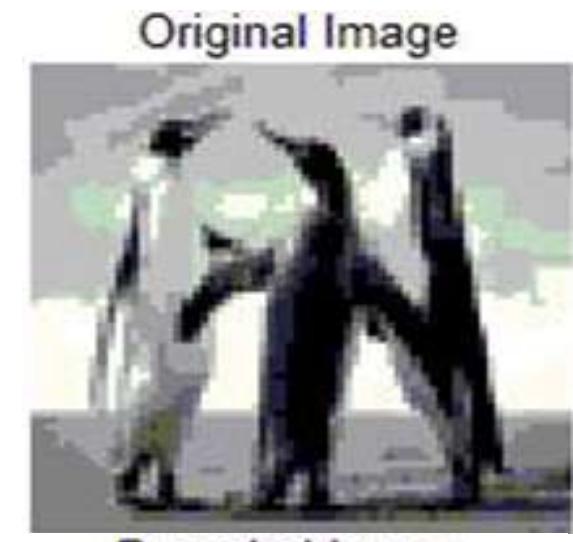

Decoded Image

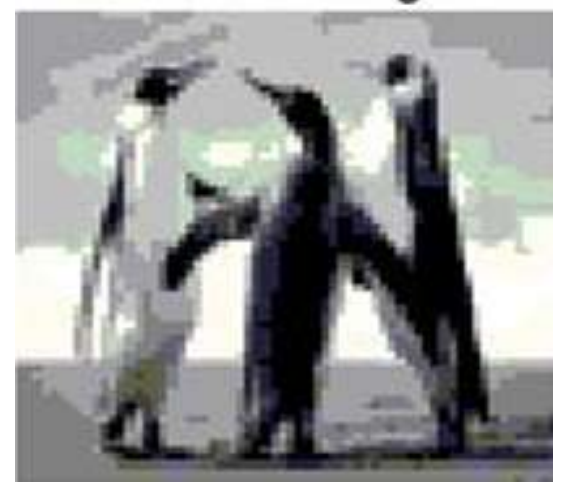

Figure 6: Processed Input and Output Images

Table 1: Comparison table for BER at 30dB SNR

\begin{tabular}{|c|c|c|}
\hline \multirow{2}{*}{ Image Input } & BER for proposed & BER for single \\
\cline { 2 - 3 } & MIMO system & OFDM transmitter \\
\hline Penguin & $1.2 \times 10^{-4}$ & $2.2 \times 10^{-3}$ \\
\hline Desert & $1.7 \times 10^{-4}$ & $3.9 \times 10^{-3}$ \\
\hline Flower & $1.5 \times 10^{-4}$ & $3.5 \times 10^{-3}$ \\
\hline Jelly fish & $1.1 \times 10^{-4}$ & $3.1 \times 10^{-3}$ \\
\hline Light house & $1.2 \times 10^{-4}$ & $1.7 \times 10^{-3}$ \\
\hline Koala & $1.4 \times 10^{-4}$ & $2.6 \times 10^{-3}$ \\
\hline Water lilies & $1.6 \times 10^{-4}$ & $2.9 \times 10^{-3}$ \\
\hline Sunset & $1.8 \times 10^{-4}$ & $2.5 \times 10^{-3}$ \\
\hline
\end{tabular}

\section{Conclusion}

In this paper, we introduced an unequal power allotment plot for the transmission of packed pictures over MIMO frameworks over an uproarious channel. The proposed framework utilizes OFDM and disorganized encoder plans. The information is gone through disorganized encoder and OFDM transmitter and is transmitted utilizing MIMO design. At the less than desirable end turn around is taken after and afterward the mean of the got picture is figured to give the yield picture. The disorderly encoder guarantees secure picture transmission and the utilization of two separate transmitters decreases the channel bending. The proposed framework is tried utilizing different pictures. The outcomes demonstrate that the proposed framework has less postponement and less BER for a given SNR.

\section{References}

[1] G. Caire and S. Shamai (Shitz), "On the Achievable Throughput of a Multi-antenna Gaussian Broadcast Channel," IEEE Trans. Inform. Theory, vol. 49, no. 7, pp. 1691-1706, Jul. 2003.

[2] H. Yang, "A road to future broadband wireless access: MIMO-OFDM-Based air interface," IEEE Communication. Mag., vol. 43, no. 1, pp. 53-60, Jan. 2005.

[3] Kellert, Stephen H., "In the Wake of Chaos: Unpredictable Order in Dynamical Systems", University of Chicago Press, ISBN 0-226-42976-8, 1993.

[4] K.M. Cuomo, A.V. Oppenheim and S. H. Strogatz, "Synchronisation of lorenz-based chaotic circuits with application to communications", IEEE Transactions on Circuits and Systems I: Fundamental Theory and Application, 626-659, 1993.

[5] M. Hasler, "Synchronization of chaotic systems and transmission of information", International Journal of Bifurcation Chaos, 647-661, 1998

[6] M. Chen, and W. Min, "Unknown input observer based chaotic secure communication", Physics Letters A, p.p. 1595-1600, 2007.

[7] L. Kocarev, K. S. Halle, K. Eckert, L. O. Chua, and U. Parlitz, "Experimental demonstration of secure communications via chaotic synchronization," International Journal of Bifurcation and Chaos, vol. 2, pp. 709-713, 1992.

\section{Volume 6 Issue 12, December 2017}




\section{International Journal of Science and Research (IJSR) \\ ISSN (Online): 2319-7064}

Index Copernicus Value (2016): 79.57 | Impact Factor (2015): 6.391

[8] Z. Li and D. Xu, "A secure communication scheme using projective chaos synchronization," Chaos, Solitons \& Fractals, vol. 22, pp. 477-481, 2004.

[9] J. Y. Chen, K. W. Wong, L. M. Cheng, and J. W. Shuai, "A secure communication scheme on the basis of the phase synchronization of chaotic systems," Chaos, vol. 13, pp. 508-514, 2003.

[10]Tsun-I Chien and Teh-Lu Liao, "Design of secure digital communication systems using chaotic modulation, cryptography and chaotic synchronization", Chaos, Solitons and Fractals 24 (2005) 241- 255

[11] T. Yang, C. W. Wu, and L. O. Chua, "Cryptography centered on chaotic systems," IEEE Transactions on Circuits and Systems-I: Fundamental Theory and Applications, vol. 44, pp. 469-472, 1997.

[12]Hang-Hong Kuo, Teh-Lu Liao, Jun-Juh Yan and JuiSheng Lin, "A fresh structure of chaotic secure communication in wireless AWGN channel", International Workshop on Chaos-Fractals Theories and Applications, 2009.

Volume 6 Issue 12, December 2017

www.ijsr.net

Licensed Under Creative Commons Attribution CC BY 